\title{
A Renewal-Theoretical Framework for Dynamic Spectrum Access with Unknown Primary Behavior
}

\author{
Chunxiao Jiang*†, Yan Chen*, and K. J. Ray Liu* \\ * Department of Electrical and Computer Engineering, University of Maryland, College Park, MD 20742, USA \\ ${ }^{\dagger}$ Tsinghua National Laboratory for Information Science and Technology (TNList) \\ Department of Electronic Engineering, Tsinghua University, Beijing 100084, P. R. China \\ E-mail:\{jcx, yan, kjrliu\}@umd.edu
}

\begin{abstract}
Dynamic spectrum access in cognitive radio networks can greatly improve the spectrum utilization efficiency. Nevertheless, interference may be introduced to the Primary User (PU) when the Secondary Users (SUs) dynamically utilize the licensed channel. If the SUs can be synchronous with the PUs, the interference is mainly due to their imperfect spectrum sensing of the primary channel. However, if the SUs have no knowledge about the PU's communication mechanism, additional interference may occur. In this paper, we propose a renewal theoretical framework to study the situation when SUs confronting with unknown primary behavior. We quantify the interference caused by the SUs and derive the corresponding close-form expressions. With the interference analysis, we study how to optimize the SUs' performance under the constraints of the PU's communication quality of service $(\mathrm{QoS})$. Finally, simulation results are shown to verify the effectiveness of our analysis.
\end{abstract}

\section{INTRODUCTION}

With the development of new wireless applications and devices, available electromagnetic radio spectrums are becoming more and more crowded. Compared with static spectrum allocation, dynamic spectrum access (DSA) technology of cognitive radio networks can greatly enhance the utilization efficiency of the existing spectrum resources [1]. The essence of DSA technology is that devices with cognitive capability, called as Secondary Users (SUs), can dynamically utilize the licensed spectrum resources that not occupied by the licensed users, usually called as Primary Users (PUs) [2].

In DSA, one of the most important issue for the SUs is how to access the primary channel while guarantee the PUs' communication quality of service (QoS), i.e., controlling the SUs' interference level. Lots of works have been done concerning the situation when the primary network is slotted and the SUs are synchronous with the PUs, including the partially observable Markov decision process model in [3], the evolutionary game model in [4] and the queuing theoretical model in [5]. In such synchronous scenario, the SUs perform spectrum sensing at the beginning of each slot, and vacate the primary channel by the end of the slot. In such a case, the potential interference is only from their imperfect spectrum sensing. The main task for the SUs is to improve the spectrum sensing performance to enhance the detection probability [6].

However, if the SUs have no knowledge about the exact time table in the primary network, those synchronous schemes will not work. On one hand, there is no concept of "time slot" for the SUs anymore. On the other hand, the SUs may fail to discover the PU's recurrence during their access time and additional interference will appear besides interference from imperfect spectrum sensing. Considering these problems, we propose a renewal theoretical framework to analyze the situation when the SUs are confronted with unknown primary behavior. We show that the SUs' dynamic behavior in the primary channel is a renewal process and quantify the interference caused by the SUs' behavior. To simplify the analysis and give more insights into the interference analysis, we assumed perfect spectrum sensing in this paper. Moreover, we formulate an optimization problem to control the SUs' dynamic access time, where the objective function is to maximize the SUs' average data rate with the constraint that the PU's average data rate should not be lower than a pre-determined threshold.

There are some works using renewal theory for cognitive radio networks. In [7], the primary channel was modeled as an ON-OFF renewal process to study how to discover spectrum holes through adjusting the SUs' sensing period. As the extension works of [7], Xue et. al. designed a periodical MAC protocol for the SUs in [8], while Tang and Chew analyzed the periodical sensing errors in [9]. In [10][11], the authors discussed how to efficiently perform channel access and switch according to the residual time of the ON-OFF process in the primary channel. Based on the assumption that the primary channel is an ON-OFF renewal process, the delay performance of the SUs were analyzed in [12][13]. However, all these related works have only modeled the PU's behavior in the primary channel as an ON-OFF process. In this paper, we further show and study the renewal characteristic of the SUs' communication behavior and analyze the interference to the PU when they dynamically access the primary channel.

The rest of this paper is organized as follows. Firstly, our system model is described in Section II. Then, we analyze the SUs' behavior in III and the interference to the PUs in Section IV. In Section V, we discuss how to optimize the SUs' performance. Finally, simulation results are shown in Section VI and conclusion is drawn in Section VII.

\section{SYSTEM MODEL}

\section{A. Network Entity}

We consider a cognitive radio network with one PU and a group SUs operating on one primary channel. An important 


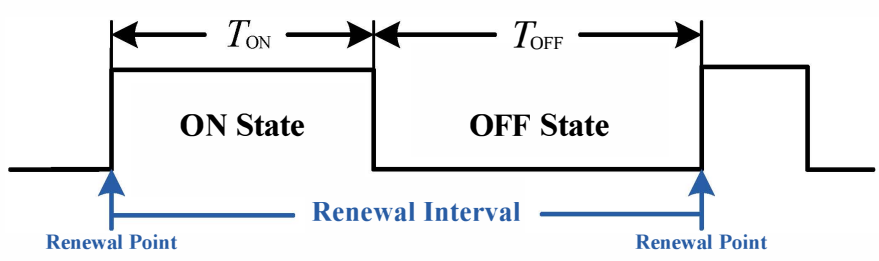

Fig. 1. Illustration of the ON-OFF primary channel state.

feature of our system is that the communication mechanism in the primary network is private, i.e., the SUs have no knowledge when the PU's communication will arrive. Moreover, we assume that the SUs are half-duplex, which means they cannot simultaneously transmit packet and perform spectrum sensing. We also assume that as long as there are available primary channels, the SUs will access these available channels. This assumption means that we are analyzing the worst case, or the maximum interference is considered since SUs are always trying to access primary channels.

\section{B. Primary Channel State Model}

Since the SUs have no idea about the exact communication mechanism of the primary network and hence cannot be synchronous with the PU, there is no concept of "time slot" in the primary channel from the SUs' points of view. Instead, the primary channel just alternatively switches between ON state and OFF state, as shown in Fig. 1.

We model the length of the ON state and OFF state by two random variables $T_{\mathrm{ON}}$ and $T_{\mathrm{OFF}}$ respectively. According to different types of the primary services (e.g., digital TV broadcasting or cellular communication), $T_{\mathrm{ON}}$ and $T_{\mathrm{OFF}}$ statistically satisfy different distributions. In this paper, we assume that $T_{\mathrm{oN}}$ and $T_{\text {OFF }}$ are independent and satisfy exponential distributions with parameter $\lambda_{1}$ and $\lambda_{0}$ respectively, denoted by $f_{\mathrm{ov}}(t)$ and $f_{\text {OFF }}(t)$ as follows:

$$
\left\{\begin{array}{l}
T_{\mathrm{ON}} \sim f_{\mathrm{ON}}(t)=\frac{1}{\lambda_{1}} e^{-t / \lambda_{1}}, \\
T_{\mathrm{OFF}} \sim f_{\mathrm{OFF}}(t)=\frac{1}{\lambda_{0}} e^{-t / \lambda_{0}} .
\end{array}\right.
$$

In such a case, the expected lengths of the ON state and OFF state are $\lambda_{1}$ and $\lambda_{0}$ accordingly. These two important parameters $\lambda_{1}$ and $\lambda_{0}$ can be effectively estimated by a maximum likelihood estimator [7]. Such an ON-OFF behavior of the PU is a combination of two Poisson process, which is a renewal process [14]. The renewal interval is $T_{p}=T_{\mathrm{ON}}+T_{\mathrm{OFF}}$ and the distribution of $T_{p}$, denoted by $f_{p}(t)$, is

$$
f_{p}(t)=f_{\mathrm{ON}}(t) * f_{\mathrm{OFF}}(t),
$$

where the symbol "*" represents the convolution operation.

\section{SUs' COMmUNiCATION BEHAVIOR ANALYSIS}

Since the SUs are always trying to access the primary channel, they are either transmitting packets or waiting for the OFF state of the primary channel. As shown in Fig. 2, the SUs' behavior dynamically switches between transmitting packets and waiting for the OFF state. The waiting time, denoted

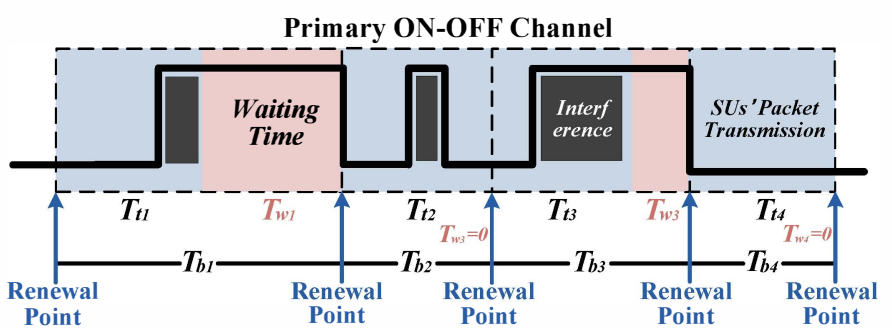

Fig. 2. SUs' renewal process in the primary channel.

by $T_{w}$, will appear if the previous transmission ends in the ON state, and the value of $T_{w}$ is determined by the length of the remaining time in the primary channel's ON state. In the following, we will analyze the SUs' communication behavior based on the renewal theory.

Theorem 1: When the SUs are always intent to access the primary channel, their communication behavior is a renewal process in the primary channel.

Proof: As shown in Fig. 2, we use $T_{b}$ to denote the interval of two adjacent transmission beginnings, i.e., $T_{b}=T_{t}+T_{w}$, where $T_{t}$ is the transmission time and $T_{w}$ is the waiting time. According to the renewal theory [14], the SUs' communication behavior is a renewal process if and only if $T_{b 1}, T_{b 2}, \ldots$ is a sequence of positive independent identically distributed (i.i.d) random variables. Since the packet transmission time $T_{t}$ is a fixed constant, Theorem 1 will hold as long as we can prove that all $T_{w 1}, T_{w 2} \ldots$ are i.i.d.

On one hand, if $T_{t i}$ ends in the OFF state, the following waiting time $T_{w i}$ will be 0 , such as $T_{w 2}$ and $T_{w 4}$ in Fig. 2. On the other hand, if $T_{t i}$ ends in the ON state, the length of $T_{w i}$ will depend on when this ON state terminates, which can be specifically illustrated in Fig. 3. In the second case, according to the renewal theory [14], $T_{w}$ is equivalent to the forward recurrence time of the ON state, $\widehat{T}_{\mathrm{ON}}$, the distribution of which is only related to that of the ON state. Thus, we can summarize $T_{w i}$ as follows

$$
T_{w i}= \begin{cases}0 & T_{t i} \text { ends in the OFF state, } \\ \widehat{T}_{\mathrm{ON} i} & T_{t i} \text { ends in the ON state. }\end{cases}
$$

From (3), it can be seen that all $T_{w i} \mathrm{~s}$ are identically distributed. Meanwhile, since each $T_{w i}$ is only determined by the corresponding $T_{t i}$ and $\widehat{T}_{\mathrm{ON}}$, all $T_{w i}$ s are independent with each other. Thus, the sequence of the waiting time $T_{w 1}, T_{w 2} \ldots$ are i.i.d, which means all $T_{b 1}, T_{b 2} \ldots$ are also i.i.d. Therefore, the SUs' communication behavior is a renewal process.

\section{SUS' INTERFERENCE ANALYSIS}

In this section, we will discuss the interference to the PU. The shaded regions in Fig. 2. indicate the interference periods in the ON state of the primary channel. In order to illustrate the impacts of these interference periods on the PU, we define the interference quantity $Q_{I}$ as follows.

Definition 1: The interference quantity $Q_{I}$ is the proportion of accumulated interference periods to the length of all $\mathrm{ON}$ 
states in the primary channel within a long period time, which can be written by

$$
Q_{I}=\lim _{T \rightarrow+\infty} \frac{\sum_{T} \text { Interference periods }}{\sum_{T} T_{\mathrm{ON}}} .
$$

In order to analyze the interference during the SUs' one packet transmission time $T_{t}$, we first introduce a new function, $I(t)$, defined as follows.

Definition 2: $I(t)$ is the expected accumulated interference to the PU within a period of time $t$, where $t$ has two special characteristics listed as follows:

- period $t$ begins at the OFF state of the primary channel,

- during $t$, the SUs keep transmitting packets.

According to Definition 1, Definition 2 and Theorem 1, the interference quantity $Q_{I}$ can be calculated by

$$
Q_{I}=\frac{I\left(T_{t}\right)}{I\left(T_{t}\right)+\mathbb{E}\left(T_{w}\right)},
$$

where $I\left(T_{t}\right)$ is the expected interference generated during the SUs' transmission time $T_{t}, \mathbb{E}\left(T_{w}\right)$ is the expectation of SUs' waiting time $T_{w}$. In the following, we will derive the closeform expressions for $I\left(T_{t}\right)$ and $\mathbb{E}\left(T_{w}\right)$ respectively.

\section{A. Expected Interference $I\left(T_{t}\right)$}

According to Definition 2, $I(t)$ is the expected length of all ON states within a period of time $t$, given that $t$ begins at the OFF state. According to the renewal theory [14], the PU's ONOFF behavior is a renewal process. Therefore, we can derive $I(t)$ through solving the renewal equation (6) according to the following Theorem 2.

Theorem 2: $I(t)$ satisfies the renewal equation as follows

$$
I(t)=\lambda_{1} F_{p}(t)+\int_{0}^{t} I(t-w) f_{p}(w) d w,
$$

where $f_{p}(t)$ is the p.d.f of the PU's renewal interval given in (2) and $F_{p}(t)$ is the corresponding cumulative distribution function (c.d.f).

Proof: Let $X$ denote the first OFF state and $Y$ denote the first ON state, as shown in Fig. 4. Thus, we can write the recursive expression of function $I(t)$ as follows:

$$
I(t)= \begin{cases}0 & t \leq X, \\ t-X & X \leq t \leq X+Y, \\ Y+I(t-X-Y) & X+Y \leq t\end{cases}
$$

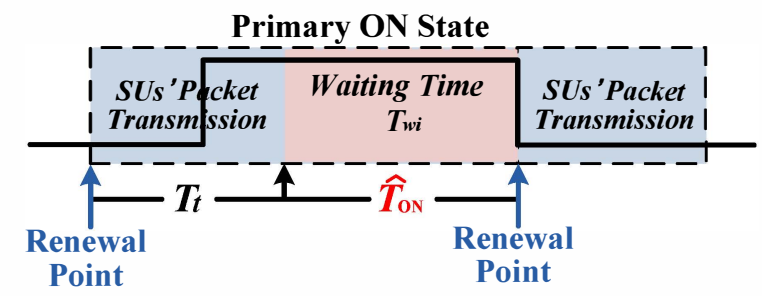

Fig. 3. SUs' waiting time $T_{w}$.

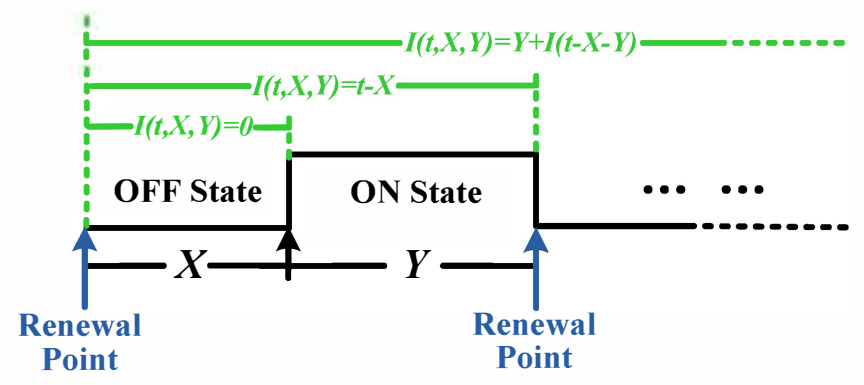

Fig. 4. Illustration of function $I(t)$.

where $X \sim f_{\text {OFF }}(x)=\frac{1}{\lambda_{0}} e^{-x / \lambda_{\bullet}}$ and $Y \sim f_{\text {oN }}(y)=\frac{1}{\lambda_{1}} e^{-y / \lambda_{1}}$.

Since $X$ and $Y$ are independent, their joint distribution $f_{X Y}(x, y)=f_{\text {OFF }}(x) f_{\text {ON }}(y)$. In such a case, $I(t)$ can be rewritten as follows:

$$
\begin{aligned}
I(t)= & \iint_{x \leq t \leq x+y}(t-x) f_{X Y}(x, y) d x d y+ \\
& \iint_{x+y \leq t}[y+I(t-x-y)] f_{X Y}(x, y) d x d y \\
= & \int_{0}^{t}(t-x) f_{\mathrm{OFF}}(x) d x+ \\
& \int_{x+y \leq t} I(t-x-y) f_{\mathrm{OFF}}(x) f_{\mathrm{ON}}(y) d x d y- \\
& \iint_{x+y \leq t}(t-x-y) f_{\mathrm{OFF}}(x) f_{\mathrm{ON}}(y) d x d y \\
= & I_{1}(t)+I_{2}(t)-I_{3}(t),
\end{aligned}
$$

where $I_{1}(t), I_{2}(t)$ and $I_{3}(t)$ represent those three terms in the second equality, respectively. By taking Laplace transforms on the both sides of (8), we have

$$
\mathbb{I}(s)=\mathbb{I}_{1}(s)+\mathbb{I}_{2}(s)-\mathbb{I}_{3}(s),
$$

where $\mathbb{I}_{1}(s), \mathbb{I}_{2}(s), \mathbb{I}_{3}(s)$ are the Laplace transforms of $I_{1}(t)$, $I_{2}(t), I_{3}(t)$, respectively.

According to the expression of $I_{1}(t)$ in (8), we have

$$
I_{1}(t)=\int_{0}^{t}(t-x) f_{\mathrm{OFF}}(x) d x=t * f_{\mathrm{OFF}}(t) .
$$

Thus, the Laplace transform of $I_{1}(t), \mathbb{I}_{1}(s)$ is

$$
\mathbb{I}_{1}(s)=\frac{1}{s^{2}} \mathbb{F}_{\mathrm{OFF}}(s),
$$

where $\mathbb{F}_{\mathrm{OFF}}(s)=\frac{1}{\lambda_{0} s+1}$ is the Laplace transform of $f_{\mathrm{OFF}}(t)$.

With the expression of $I_{2}(t)$ in (8), we have

$$
\begin{aligned}
I_{2}(t) & =\iint_{x+y \leq t} I(t-x-y) f_{\mathrm{OFF}}(x) f_{\mathrm{ON}}(y) d x d y \\
& =I(t) * f_{\mathrm{ON}}(t) * f_{\mathrm{OFF}}(t)=I(t) * f_{p}(t),
\end{aligned}
$$

where the last step is according to (2). Thus, the Laplace transform of $I_{2}(t), \mathbb{I}_{2}(s)$ is

$$
\mathbb{I}_{2}(s)=\mathbb{I}(s) \mathbb{F}_{p}(s),
$$


where $\mathbb{I}(s)$ and $\mathbb{F}_{p}(s)=\frac{1}{\left(\lambda_{1} s+1\right)\left(\lambda_{\bullet} s+1\right)}$ are Laplace transforms of $I(t)$ and $f_{p}(t)$, respectively.

Similar to (12), we can re-written $I_{3}(t)$ as $I_{3}(t)=t * f_{p}(t)$. Thus, the Laplace transform of $I_{3}(t), \mathbb{I}_{3}(s)$ is

$$
\mathbb{I}_{3}(s)=\frac{1}{s^{2}} \mathbb{F}_{p}(s) .
$$

By substituting (11), (13) and (14) into (9), we have

$$
\begin{aligned}
\mathbb{I}(s) & =\frac{1}{s^{2}} \mathbb{F}_{\mathrm{OFF}}(s)+\mathbb{I}(s) \mathbb{F}_{p}(s)-\frac{1}{s^{2}} \mathbb{F}_{p}(s) \\
& =\lambda_{1} \frac{\mathbb{F}_{p}(s)}{s}+\mathbb{I}(s) \mathbb{F}_{p}(s) .
\end{aligned}
$$

Then by taking the inverse Laplace transform on (15), we have

$$
\begin{aligned}
I(t) & =\lambda_{1} \int_{0}^{t} f_{p}(w) d w+\int_{0}^{t} I(t-w) f_{p}(w) d w \\
& =\lambda_{1} F_{p}(t)+\int_{0}^{t} I(t-w) f_{p}(w) d w .
\end{aligned}
$$

This completes the proof of the theorem.

Theorem 2 illustrates the renewal characteristic of $I(t)$. By substituting $\mathbb{F}_{p}(s)=\frac{1}{\left(\lambda_{1} s+1\right)\left(\lambda_{\bullet} s+1\right)}$ into (15), the Laplace transform of $I(t)$ can be calculated by

$$
\mathbb{I}(s)=\frac{\lambda_{1} \mathbb{F}_{p}(s)}{s\left(1-\mathbb{F}_{p}(s)\right)}=\frac{\lambda_{1}}{s^{2}\left(\lambda_{0} \lambda_{1} s+\lambda_{0}+\lambda_{1}\right)} .
$$

Then, by taking inverse Laplace transform on (17), we can obtain the close-form expression for $I(t)$ as

$$
I(t)=\frac{\lambda_{1}}{\lambda_{0}+\lambda_{1}} t-\frac{\lambda_{0} \lambda_{1}^{2}}{\left(\lambda_{0}+\lambda_{1}\right)^{2}}\left(1-e^{-\frac{\lambda_{\bullet}+\lambda_{1}}{\lambda_{\bullet} \lambda_{1}} t}\right) .
$$

\section{B. Expected Waiting Time $\mathbb{E}\left(T_{w}\right)$}

The definition of waiting time $T_{w}$ has been given in (3) in the proof of Theorem 1. To compute the expected waiting time, we introduce a new function defined as follows.

Definition 3: $P_{\mathrm{ON}}(t)$ is the average probability that a period of time $t$ begins at the OFF state and ends at the ON state.

According to Definition 3 and (3), the SUs' average waiting time $\mathbb{E}\left(T_{w}\right)$ can be written by

$$
\mathbb{E}\left(T_{w}\right)=P_{\mathrm{ON}}\left(T_{t}\right) \cdot \mathbb{E}\left(\widehat{T}_{\mathrm{ON}}\right) .
$$

In the following, we will derive the close-form expressions for $P_{\mathrm{ON}}\left(T_{t}\right)$ and $\mathbb{E}\left(\widehat{T}_{\mathrm{ON}}\right)$, respectively.

Similar to the analysis of $I(t)$ in Section IV-A, $P_{\mathrm{ON}}(t)$ can also be obtained through solving the renewal equation (20) according to the following Theorem 3 .

Theorem 3: $P_{\mathrm{ON}}(t)$ satisfies the renewal equation as

$$
P_{\mathrm{ON}}(t)=\lambda_{1} f_{p}(t)+\int_{0}^{t} P_{\mathrm{ON}}(t-w) f_{p}(w) d w .
$$

Proof: Similar to $I(t)$ in (7), the recursive expression of $P_{\mathrm{ON}}(t)$ can be written by

$$
P_{\mathrm{ON}}(t)= \begin{cases}0 & t \leq X, \\ 1 & X \leq t \leq X+Y, \\ P_{\mathrm{ON}}(t-X-Y) & X+Y \leq t .\end{cases}
$$

where $X$ and $Y$ are same with those in (7). In such a case, $P_{\mathrm{ON}}(t)$ can be re-written by

$$
\begin{aligned}
P_{\mathrm{ON}}(t)= & \iint_{x \leq t \leq x+y} f_{X Y}(x, y) d x d y+ \\
& \iint_{x+y \leq t} P_{\mathrm{ON}}(t-x-y) f_{X Y}(x, y) d x d y \\
= & F_{\mathrm{OFF}}(t)-f_{\mathrm{OFF}}(t) * F_{\mathrm{ON}}(t)+P_{\mathrm{ON}}(t) * f_{p}(t) .
\end{aligned}
$$

By taking Laplace transform on (22), we have

$$
\mathbb{P}_{\mathrm{ON}}(s)=\lambda_{1} \mathbb{F}_{\mathrm{ON}}(s) * \mathbb{F}_{p}(s)+\mathbb{P}_{\mathrm{ON}}(s) * \mathbb{F}_{p}(s) .
$$

Then by taking the inverse Laplace transform on (23), we have

$$
P_{\mathrm{ON}}(t)=\lambda_{1} f_{p}(t)+\int_{0}^{t} P_{\mathrm{ON}}(t-w) f_{p}(w) d w .
$$

This completes the proof of the theorem.

Similar to the solution to renewal equation (6) in Section IV-A, we can obtain the close-form expression of $P_{\mathrm{ON}}(t)$ through solving (24) as

$$
P_{\mathrm{ON}}(t)=\frac{\lambda_{1}}{\lambda_{0}+\lambda_{1}}\left(1-e^{-\frac{\lambda_{\bullet}+\lambda_{1}}{\lambda_{\bullet} \lambda_{1}} t}\right)
$$

The $\widehat{T}_{\mathrm{ON}}$ is the forward recurrence time of the primary channel's ON state. Since all ON sates follow a Poisson process. According to renewal theory [14], we have

$$
\widehat{T}_{\mathrm{ON}} \sim \frac{1}{\lambda_{1}} e^{-t / \lambda_{1}}, \quad \mathbb{E}\left(\widehat{T}_{\mathrm{ON}}\right)=\lambda_{1}
$$

By combining (25) and (26), the SUs' average waiting time $\mathbb{E}\left(T_{w}\right)$ can be obtained as

$$
\mathbb{E}\left(T_{w}\right)=\frac{\lambda_{1}^{2}}{\lambda_{0}+\lambda_{1}}\left(1-e^{-\frac{\lambda_{\bullet}+\lambda_{1}}{\lambda_{\bullet} \lambda_{1}} T_{t}}\right) .
$$

Finally, by substituting (18) and (27) into (5), we can obtain the quantity of interference $Q_{I}$ as

$$
Q_{I}=\frac{\left(\lambda_{0}+\lambda_{1}\right) T_{t}-\lambda_{0} \lambda_{1}\left(1-e^{-\frac{\lambda_{\bullet}+\lambda_{1}}{\lambda_{\bullet} \lambda_{1}} T_{t}}\right)}{\left(\lambda_{0}+\lambda_{1}\right) T_{t}+\lambda_{1}^{2}\left(1-e^{-\frac{\lambda_{\bullet}+\lambda_{1}}{\lambda_{\bullet} \lambda_{1}} T_{t}}\right)} .
$$

\section{Optimizing SUs' Communication PERformance}

In this section, we will discuss how to optimize the SUs' communication performance while maintaining the PU's communication QoS according to the interference analysis. In our system, the SUs' communication performance is directly dependent on the SUs' transmission time $T_{t}$. Obviously, a longer access time $T_{t}$ can help SUs achieve higher average data rate. However, a longer $T_{t}$ cam also bring more interference to PUs. In the following, we will construct an optimization problem to find the optimal $T_{t}$ for the $\mathrm{SU}$, where the objective function is to maximize the SUs' average data rate $R_{s}$ with the constraint that the PU's average data rate $R_{p}$ should not be lower than the pre-determined threshold $R_{p}^{\downarrow}$, as follows:

$$
\begin{aligned}
\max . & R_{s} \\
\text { s.t. } & R_{p} \geq R_{p}^{\downarrow} \text {. }
\end{aligned}
$$




\section{A. PUs' Average Data Rate}

If there is no interference from the SUs, the PU's instantaneous rate is $\log \left(1+\mathrm{SNR}_{p}\right)$, where $\mathrm{SNR}_{p}$ denotes the Signalto-Noise Ratio of primary signal at the PU. On the other hand, if the interference occurs, the PU's instantaneous rate will be $\log \left(1+\frac{\mathrm{SNR}_{p}}{\mathrm{INR}_{p}+1}\right)$, where $\mathrm{INR}_{p}$ is the Interference-toNoise Ratio of secondary signal received by the PU. According to Definition $1, Q_{I}$ represents the ratio of the interference periods to the PU's overall communication time. Thus, the PU's average data rate $R_{p}$ can be calculated by

$$
R_{p}=\left(1-Q_{I}\right) \log \left(1+\mathrm{SNR}_{p}\right)+Q_{I} \log \left(1+\frac{\mathrm{SNR}_{p}}{\mathrm{INR}_{p}+1}\right) .
$$

\section{B. SUs' Average Data Rate}

If a SU encounters the PU's recurrence, i.e., the ON state of the primary channel, during its transmission time $T_{t}$, its communication is also interfered by the PU's signal. In such a case, the SU's instantaneous rate is $\log \left(1+\frac{\mathrm{SNR}_{s}}{\mathrm{INR}+1}\right)$, where $\mathrm{SNR}_{s}$ is the SU's Signal-to-Noise Ratio and $\mathrm{INR}_{s}$ is the Interference-to-Noise Ratio of primary signal received by the SU. According to Theorem 1, the occurrence probability of such a phenomenon is $\frac{I\left(T_{t}\right)}{T_{t}+\mathbb{E}\left(T_{w}\right)}$. On the other hand, if no PU appears during the SU's transmission, its instantaneous rate is $\log \left(1+\mathrm{SNR}_{s}\right)$ and the corresponding occurrence probability is $\frac{T_{t}-I\left(T_{t}\right)}{T_{t}+\mathbb{E}\left(T_{w}\right)}$. Thus, the SU's average data rate $R_{s}$ is

$$
\begin{aligned}
R_{s}= & \frac{T_{t}-I\left(T_{t}\right)}{T_{t}+\mathbb{E}\left(T_{w}\right)} \cdot \log \left(1+\mathrm{SNR}_{s}\right)+ \\
& \frac{I\left(T_{t}\right)}{T_{t}+\mathbb{E}\left(T_{w}\right)} \cdot \log \left(1+\frac{\mathrm{SNR}_{s}}{\mathrm{INR}_{s}+1}\right) .
\end{aligned}
$$

Theorem 4: The PU's average data rate $R_{p}$ is a strictly decreasing function in terms of $T_{t}$ and the SUs' average data rate $R_{s}$ is a strictly increasing function in terms of $T_{t}$, i.e.,

$$
\frac{\partial R_{p}}{\partial T_{t}}<0, \quad \frac{\partial R_{s}}{\partial T_{t}}>0
$$

Proof: For simplification, we use $R_{p 0}$ to express $\log (1+$ $\left.\mathrm{SNR}_{p}\right)$ and $R_{p 1}$ to express $\log \left(1+\frac{\mathrm{SNR}_{p}}{\mathrm{INR}_{p}+1}\right)$. According to (30), $\frac{\partial R_{\boldsymbol{p}}}{\partial T_{t}}$ can be calculated as follows

$$
\frac{\partial R_{p}}{\partial T_{t}}=-\frac{\partial Q_{I}}{\partial T_{t}}\left(R_{p 0}-R_{p 1}\right)
$$

According to (28), we have

$$
\frac{\partial Q_{I}}{\partial T_{t}}=\frac{\partial Q_{I}}{\partial Y} \frac{\partial Y}{\partial T_{t}},
$$

where $Y=\frac{1-e^{-\frac{\lambda_{\bullet}+\lambda_{1}}{\lambda_{0} \lambda_{1}}} T_{t}}{T_{t}}$. Then, we have

$$
\begin{aligned}
\frac{\partial Q_{I}}{\partial Y} & =\frac{-\lambda_{1}\left(\lambda_{0}+\lambda_{1}\right)^{2}}{\left(\lambda_{0}+\lambda_{1}+\lambda_{1}^{2} Y\right)^{2}}<0, \\
\frac{\partial Y}{\partial T_{t}} & =\frac{\left(\frac{\lambda_{0}+\lambda_{1}}{\lambda_{0} \lambda_{1}} T_{t}+1\right) e^{-\frac{\lambda_{0}+\lambda_{1}}{\lambda_{0} \lambda_{1}} T_{t}}-1}{T_{t}^{2}}=\frac{g\left(T_{t}\right)-1}{T_{t}^{2}} .
\end{aligned}
$$

Since $g^{\prime}\left(T_{t}\right)<0$ and $g(0)=1$, we have $g\left(T_{t}\right)<1$ and thus $\frac{\partial Y}{\partial T_{t}}<0$. In such a case, combining (33), (34), (35) and (36), we have $\frac{\partial R_{p}}{\partial T_{t}}<0$. Similarly, through taking partial derivative on (31) in terms of $T_{t}$, we can prove that $\frac{\partial R_{s}}{\partial T_{t}}>0$, the process of which is omitted.

From Theorem 4, we can see that the objective function and the constraints are all monotonous functions in terms of $T_{t}$. Thus, the solution to the optimization problem (29) can be found using gradient descent method [15].

\section{Simulation Results}

In this section, we conduct simulations to verify the effectiveness of our analysis. The parameters of primary ON-OFF channel are set to be $\lambda_{0}=2.6 \mathrm{~s}$ and $\lambda_{1}=3.6 \mathrm{~s}$.

\section{A. Interference Quantity $Q_{I}$}

In Fig. 5, we illustrate the theoretic and simulated results of interference quantity to the PU, $Q_{I}$. The theoretic results are computed according to (28) with the SUs' transmission time $T_{t}=0.6 \mathrm{~s}$. For the simulated results, once the interference occurs, we calculate and record the ratio of the accumulated interference periods to the accumulated periods of the $\mathrm{ON}$ states. From Fig. 5, we can see that the simulated results eventually converge to the corresponding theoretic results after some fluctuations at the beginning, which means that the close-form expressions in (28) are correct and can be used to calculate the interference caused by the SUs in the practical cognitive radio system.

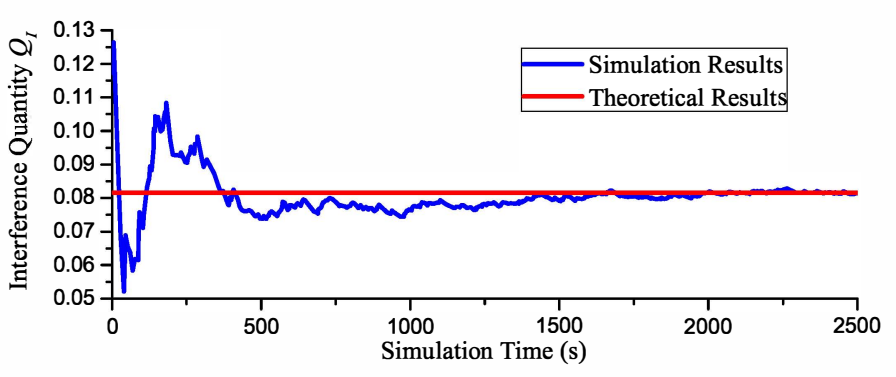

Fig. 5. Interference quantity $Q_{I}$ when $T_{t}=0.6 \mathrm{~s}$.

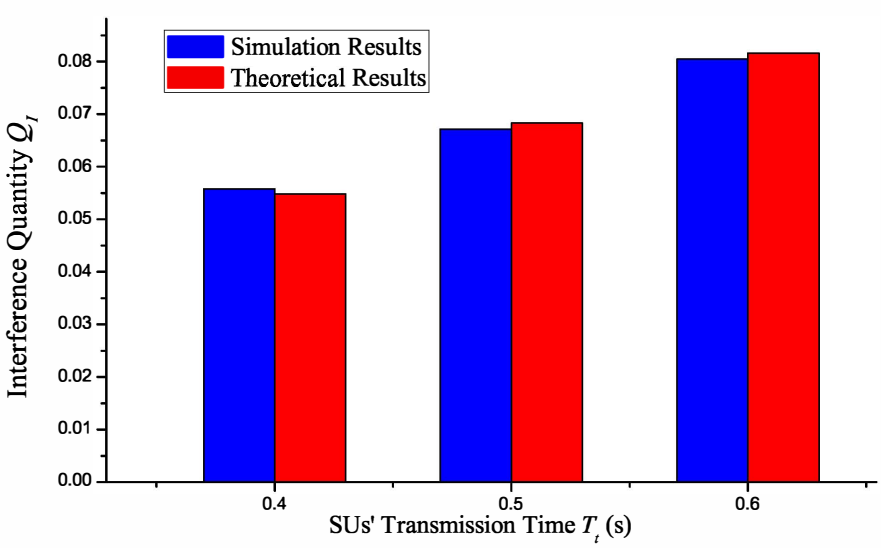

Fig. 6. Interference quantity $Q_{I}$ when $T_{t}=0.4,0.5,0.6 \mathrm{~s}$. 
In Fig. 6, we also illustrate the theoretic and simulated $Q_{I}$ when the SUs' transmission time $T_{t}=0.4,0.5,0.6 \mathrm{~s}$. We can see that the interference increases as the SUs' transmission time $T_{t}$ increases. Such a phenomenon is because the interference to the PU can only occur during $T_{t}$ and the increase of $T_{t}$ enlarges the occurrence probability of $T_{t}$.

\section{B. PU's and SUs' Average Data Rate}

The simulation results of the PU's and SUs' average data rate $R_{p}$ and $R_{s}$ versus the SUs' transmission time $T_{t}$ are shown in Fig. 7, where we set $\mathrm{SNR}_{p}=\mathrm{SNR}_{s}=5 \mathrm{db}$ and $\mathrm{INR}_{p}=\mathrm{INR}_{s}=3 \mathrm{db}$. We can see that $R_{p}$ is a decreasing function in terms of $T_{t}$, while $R_{s}$ is an increasing function in terms of $T_{t}$, which are in accordance with Theorem 4. Such a phenomenon is because an increase of $T_{t}$ will help the SUs obtain more transmission throughput, but also cause more interference and thus degrade the PU's average data rate.

In Fig. 8, we illustrate the SUs' maximized average data rate versus the PU's lowest average data $R_{p}^{\downarrow}$. The shaded area in Fig. 8 represents the SUs' achievable rate region under the constraint that the PU's QoS, i.e., $R_{p}^{\downarrow}$, should be guaranteed. Suppose that the PU's data rate should be at least $1.8 \mathrm{bps} / \mathrm{Hz}$, i.e., $R_{p}^{\downarrow}=1.8 \mathrm{bps} / \mathrm{Hz}$. In such a case, the SUs' average data rate can achieve around $0.93 \mathrm{bps} / \mathrm{Hz}$ according to Fig. 8. Moreover, for any fixed $R_{p}^{\downarrow}$, the maximized value of $T_{t}$ and $R_{s}$ are also determined by the channel parameters $\lambda_{0}$ and $\lambda_{1}$. Therefore, the SUs should dynamically adjust their communication behaviors according to the estimated channel parameters.

\section{CONCLUSION}

In this paper, we analyzed the scenario when the SUs confronted with unknown primary behavior. Based on the renewal theory, we showed that the SUs' communication behaviors in the ON-OFF primary channel is a renewal process and derived the close-form for the interference to the PU. We further discussed how to optimize the SUs' transmission time to control the level of interference to the PU. Simulation results are shown to validate our close-form expressions for the

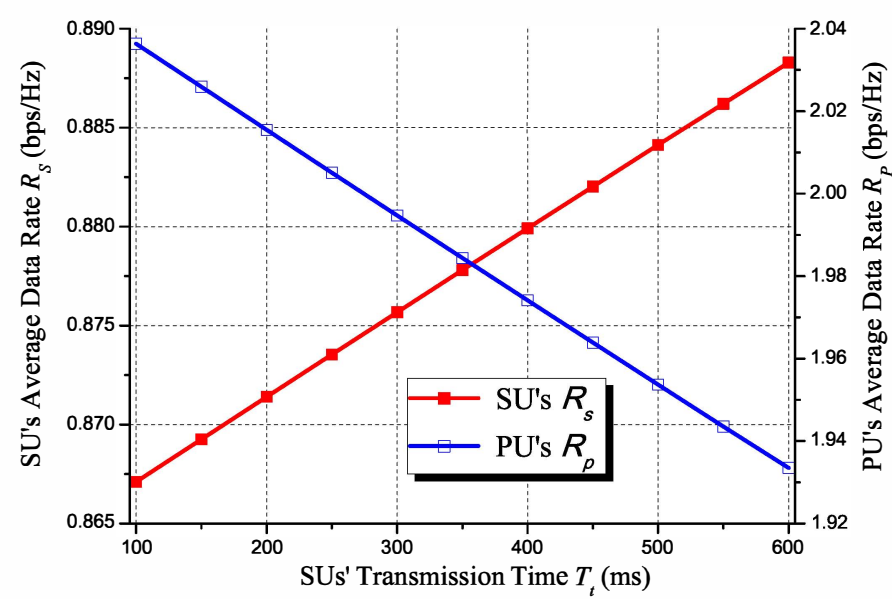

Fig. 7. PU's average data rate.

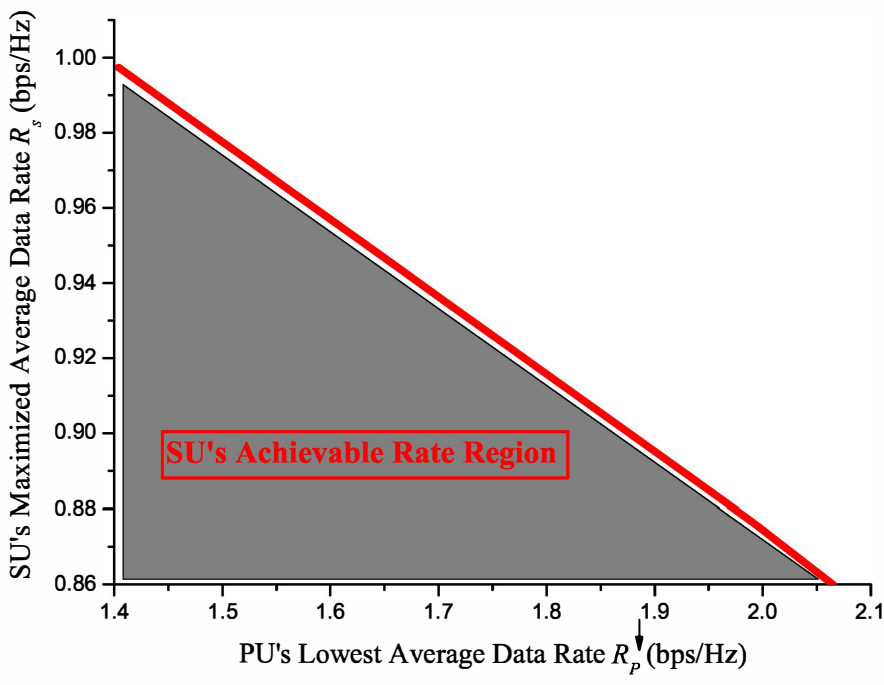

Fig. 8. SUs' average data rate.

interference quantity. In the practical cognitive radio networks, these expressions can be used to evaluate the interference from the SUs when configuring the secondary network.

\section{REFERENCES}

[1] S. Haykin, "Cognitive radio: brain-empowered wireless communications,” IEEE J. Sel. Areas Commun., vol. 23, no. 2, pp. 201-220, 2005.

[2] K. J. R. Liu and B. Wang, Cognitive Radio Networking and Security: A Game Theoretical View. Cambridge University Press, 2010.

[3] Q. Zhao, L. Tong, A. Swami, and Y. Chen, "Decentralized cognitive MAC for opportunistic spectrum access in Ad Hoc networks: a POMDP framework," IEEE J. Sel. Areas Commun., vol. 25, no. 3, pp. 589-600, 2007.

[4] B. Wang, K. J. R. Liu, and T. C. Clancy, "Evolutionary cooperative spectrum sensing game: how to collaborate?" IEEE Trans. Commun., vol. 58, no. 3, pp. 890-900, 2010.

[5] A. A. El-Sherif and K. J. R. Liu, "Joint design of spectrum sensing and channel access in cognitive radio networks," IEEE Trans. Wireless Commun., vol. 10, no. 6, pp. 1743-1753, 2011.

[6] Y.-C. Liang, Y. Zeng, E. C. Peh, and A. T. Hoang, "Sensing-throughput tradeoff for cognitive radio networks," IEEE Trans. Wireless Commun., vol. 7, no. 4, pp. 1326-1337, 2008.

[7] H. Kim and K. G. Shin, "Efficient discovery of spectrum opportunities with MAC-layer sensing in cognitive radio networks," IEEE Trans. Mobile Computing, vol. 7, no. 5, pp. 533-545, 2008.

[8] D. Xue, E. Ekici, and X. Wang, "Opportunistic periodic MAC protocol for cognitive radio networks," in Proc. IEEE GLOBECOM, 2010.

[9] P. K. Tang and Y. H. Chew, "Modeling periodic sensing errors for opportunistic spectrum access," in Proc. IEEE VTC-FALL, 2010.

[10] M. Sharma, A. Sahoo, and K. D. Nayak, "Model-based opportunistic channel access in dynamic spectrum access networks," in Proc. IEEE GLOBECOM, 2009.

[11] S. Wang, W. Wang, F. Li, and Y. Zhang, "Anticipated spectrum handover in cognitive radios," in Proc. IEEE ICT, 2011, pp. 49-54.

[12] P. Wang and I. F. Akyildiz, "On the origins of heavy tailed delay in dynamic spectrum access networks," accepted by IEEE Trans. Mobile Comput., 2011.

[13] R. Chen and X. Liu, "Delay performance of threshold policies for dynamic spectrum access," IEEE Trans. Wireless Commun., vol. 10, no. 7, pp. 2283-2293, 2011.

[14] D. R. Cox, Renewal Theory. Butler and Tanner, 1967.

[15] D. P. Bertsekas, Nonlinear Programming. Athena Scientific, 1999. 